\title{
Prenatal prediction of spinal muscular atrophy
}

\author{
R J Daniels, G K Suthers, K E Morrison, N H Thomas, M J Francis, C G Mathew, \\ $S$ Loughlin, A Heiberg, D Wood, V Dubowitz, K E Davies
}

\begin{abstract}
Spinal muscular atrophy (SMA) is a common cause of inherited morbidity and mortality in childhood. The wide range of phenotypes in SMA, uncertainty regarding its mode of inheritance, and the suggestion of linkage heterogeneity have complicated the genetic counselling of parents of affected children. The locus responsible for autosomal recessive SMA has been mapped to 5q11.2-q13.3. The most likely order of loci is cenD5S6-(SMA,D5S125)-(JK53CA1/2,D5S112)D5S39-qter, with highly polymorphic loci being identified at JK53CA1/2 and D5S39. We describe linkage studies with another highly polymorphic locus, D5S127, that is closely linked to DSS39. This genetic map can be used as the basis for genetic counselling in families with autosomal recessive SMA. Appropriate allowance can be made for sporadic cases owing to non-inherited causes and for linkage heterogeneity or misdiagnoses.
\end{abstract}

The locus for autosomal recessive spinal muscular atrophy (SMA) has been mapped to the proximal long arm of human chromosome $5 .{ }^{1-5}$ The disease primarily affects the anterior horn cells of the spinal cord and motor cranial nerve nuclei, but nothing is known of the pathogenesis. Affected subjects generally present with one of three forms of the disease. ${ }^{6}$ The most severe type (type I, acute SMA or WerdnigHoffmann disease) is characterised by onset in the first six months of life, failure to sit, and usually death by the age of 2 years. An intermediate form (type II) has a later onset but the affected child never walks unaided. The prognosis in these cases is variable and depends on the degree to which the respiratory muscles are affected. In the comparatively mild form of the disease (type III, Kugelberg-Welander disease), affected subjects maintain independent ambulation. Allelic mutations at the SMA locus are thought to account for the variation in phenotype.

We have recently localised $S M A$ in relation to highly polymorphic loci at $5 \mathrm{q} 11.2-\mathrm{q} 13.3$ in families with two or more affected persons. ${ }^{78}$ The order of loci (and sex averaged recombination fractions) are cen-D5S6-(0.02)D5S125-(0.04)-( $7 K 53 C A 1 / 2, D 5 S 112)-(0.04)-$ $D 5 S 39$-qter. No recombination was observed between $S M A$ and DSS125. The closest markers known to flank $S M A$ are D5S6 and $(\mathcal{F} K 53 C A 1 / 2, D 5 S 112)$. The distance between these loci is only $6 \mathrm{cM}$.

In view of this precise localisation of $S M A$, it should be possible to offer couples who have had an affected child prenatal diagnosis during subsequent pregnancies. However, there are a number of issues which confound the direct application of this genetic map to the counselling of affected families. First, SMA is a clinically heterogeneous disorder. ${ }^{69}$ Although the clinical features are usually consistent among the affected subjects in a pedigree, some reported pedigrees show wide variation in the severity of muscle weakness. In the absence of a clinical sign or investigation specific for SMA the wide variation in phenotype raises the possibility of misdiagnosis, particularly when evaluating an isolated case.

Second, a comprehensive genetic model of SMA has not been described. It is generally agreed that severe SMA is autosomal recessive, but a number of studies have indicated that the segregation ratio in pedigrees with intermediate or mild SMA is significantly less than the value of 0.25 expected for an autosomal recessive disorder. ${ }^{910}$ It is not known whether this distortion of the segregation ratio reflects misdiagnoses, a high mutation rate, or a complex genetic model. Conversely, some pedigrees have an unexpectedly high frequency of cases among second degree relatives which could be explained by a three allele single locus model..$^{11-13}$

Third, evidence has been presented indicating genetic heterogeneity among pedigrees with SMA of all types. ${ }^{257}$ The majority of families studied indicated linkage of $S M A$ to $D 5 S 6$, but approximately $15 \%$ were not linked to $5 \mathrm{q}$ loci. Tests of linkage heterogeneity do not distinguish between genetic heterogeneity owing to a second disease locus or apparent heterogeneity owing to the misclassification of some persons or families. A number of the unlinked families have been clinically reviewed, and the original diagnosis of SMA has been refuted. ${ }^{14}{ }^{15}$ Although there may be no linkage heterogeneity among a tightly defined group of families with SMA, the possibility of misdiagnosis remains when counselling an individual family.

In this paper we describe the genetic localisation of another highly polymorphic locus in relation to SMA, and present our approach to prenatal diagnosis in SMA families.

\section{Methods}

Restriction fragment length polymorphisms (RFLPs) have been documented at D5S6, $D 5 S 112$, and DSS39. The other polymorphisms used in this study were $(\mathrm{CA})_{\mathrm{n}}$ dinucleotide repeats. ${ }^{16}$ Details of the polymorphisms, primers, and PCR conditions are summarised in tables 1 and 2.
Received 9 September 1991 Revised version accepted 15 October 1991 . 
Table 1 Polymorphic loci closely linked to SMA.

\begin{tabular}{|c|c|c|c|c|}
\hline D No & Probe name & Type & PIC & Sizes \\
\hline $\begin{array}{l}\text { DSS6 } \\
\text { DSS125 } \\
\\
D S S 112 \\
D S S 39 \\
D 5 S 39 \\
D 5 S 127\end{array}$ & $\begin{array}{l}\text { pM4 } \\
\text { EF(TG/AG) } \\
\text { JK53CA1/2 } \\
\text { JK53 } \\
\text { 153-6741GT } \\
\text { p105-153RA } \\
\text { YN(CT) })_{n}\end{array}$ & $\begin{array}{l}\text { BamHI } \\
\mathrm{CA}_{\mathrm{n}} \\
\mathrm{CA}_{\mathrm{n}} \\
P v u I I \\
\mathrm{CA}_{\mathrm{n}} \\
M s p \mathrm{I} \\
\mathrm{CA}_{\mathrm{n}}\end{array}$ & $\begin{array}{l}0 \cdot 51 \\
0 \cdot 36 \\
0 \cdot 74 \\
0 \cdot 37 \\
0 \cdot 74 \\
0 \cdot 36 \\
0 \cdot 84\end{array}$ & $\begin{array}{l}\text { A1:11 kb; A2:9.6 kb; A3:7.6 kb } \\
143-147 \mathrm{bp} ; 2 \text { alleles } \\
94-120 \mathrm{bp} ; 12 \text { alleles } \\
\text { A1:18 kb; A2:4.9 kb } \\
212-220 \mathrm{bp} ; 5 \text { alleles } \\
\text { A1:8.7 kb; A2:5.8 kb } \\
96-114 \mathrm{bp} ; 10 \text { alleles }\end{array}$ \\
\hline
\end{tabular}

References: D5S6, ${ }^{17}$ D5S125 \& D5S $127,{ }^{18} \mathrm{JK} 53 \mathrm{CA} 1 / 2,{ }^{8}$ D5S $112,{ }^{19}$ D5S39. ${ }^{19} 20$

Linkage analysis of $D 5 S 127$ in relation to SMA and other loci was performed using genotypes from 31 pedigrees with two or more subjects having severe, intermediate, or mild SMA. The criteria for including these families in the study are published elsewhere. ${ }^{7}$ For this set of families, SMA was assumed to be a monogenic autosomal recessive disorder. The odds in favour of an autosomal recessive versus three allele model ${ }^{13}$ for this data set were $10^{8}: 1$; the genetic localisation of SMA was the same under each model. The mutant allele frequency was taken to be 0.006 , the penetrance $1 \cdot 0$, and the mutation rate zero. ${ }^{1}$

In families presenting for genetic risk counselling the diagnosis in the proband was established using defined criteria. ${ }^{6}$ The appropriate choice of genetic model is discussed below. All linkage analyses were performed using version 5.1 of the LINKAGE package of computer programs ${ }^{21}$ which makes no provision for interference when analysing three or more loci.

\section{Results}

Evaluation of the four dinucleotide repeat polymorphisms can be simplified by combining the PCR reactions for D5S125/D5S127. These four polymorphisms can also be determined from dried blood spots. Examples of the various polymorphisms at loci close to $S M A$ are shown in fig 1 .

Two point lod scores of D5S127 and the other loci are listed in table 3 , and indicate that $D 5 S 127$ is close to D5S39. The loci D5S127 and D5S39 have been co-localised to two yeast artificial chromosomes and lie within $350 \mathrm{~kb}$ of one another ( $M \mathrm{~J}$ Francis, $\mathrm{K}$ E Davies, unpublished observations). Thus, the order of loci at $5 \mathrm{q} 11-\mathrm{q} 13$ is cen-D5S6-(SMA,D5S125)$(\Im K 53 C A 1 / 2, D 5 S 112)-(D 5 S 127, D 5 S 39)$-qter.
PRENATAL COUNSELLING: CASE STUDIES

Family 1. Severe SMA (fig 2)

The proband presented at 4 months of age with delayed motor milestones and a paucity of spontaneous movement. His assessment was complicated by severe aspiration pneumonia, but a muscle biopsy was consistent with the diagnosis of SMA. He never sat unaided and died within the first year of life. The proband's parents then had monozygotic twin girls who also had severe hypotonia and died within the first year of life. There were no other sibs and there was no family history of muscle weakness. The parents presented during the third pregnancy requesting prenatal diagnosis.

The mode of inheritance of SMA was assumed to be autosomal recessive. The prior probability of the disease locus being linked to $5 \mathrm{q}$ loci in this family was arbitrarily assumed to be $95 \%$, thereby making a $5 \%$ allowance for either misdiagnosis or genetic heterogeneity. By using Bayesian analysis and the genotypes of the parents and affected children, ${ }^{223}$ the posterior probability of the family being of the linked type was $99 \%$.

After chorionic villus sampling at 10 weeks' gestation, the fetal genotype was determined at D5S6 (probe pM4) and D5S39 (probe p105153RA). Assuming the family to be of the linked type, the risk of the fetus being affected was estimated with $S M A$ located at four different points within the interval D5S6$(\mathcal{F} 53 C A 1 / 2, D 5 S 112) .{ }^{24}$ The risk interval ${ }^{25}$ was 1 to $10 \%$. Assuming the family to be of the unlinked type, the risk to the fetus would be $25 \%$. These two risk estimates were weighted according to the posterior probabilities that the family was of the linked versus unlinked type. The final risk interval for the fetus being affected was 2 to $11 \%$. The pregnancy spontaneously miscarried at 22 weeks' gestation.

\section{Family 2. Severe SMA (fig 2)}

The proband was diagnosed at 4 months of age. He had extreme hypotonia, clinically obvious fasciculation, and an EMG and muscle biopsy consistent with a diagnosis of SMA. His condition rapidly deteriorated and he died before his first birthday. He had no sibs and there was no family history of muscle weakness. The proband's parents then presented during the next pregnancy requesting prenatal diagnosis.

Table 2 PCR conditions for detecting dinucleotide repeat polymorphisms.

\begin{tabular}{|c|c|c|c|c|}
\hline Locus & Primers & Temp & $\underset{(\mathrm{mmol} / \mathrm{h})}{\mathrm{MgCl}_{2}}$ & $\begin{array}{c}\text { Time } \\
\text { (minutes) }\end{array}$ \\
\hline D5S125 & $\begin{array}{l}\text { 5'-AAGAGGACTCCCATGCTTGTTG-3' } \\
\text { 5'-TAGAAAATACTACAGTCTTCTTGGG-3' }\end{array}$ & $62^{\circ} \mathrm{C}$ & $1 \cdot 0$ & 120 \\
\hline$f K 53 C A 1 / 2$ & $\begin{array}{l}\text { 5'-TGTTCTTGGCATCACTGC-3' } \\
\text { 5'-TTTGAAGCCCTGGAATAT-3' }\end{array}$ & $54^{\circ} \mathrm{C}$ & 1.5 & 240 \\
\hline$D 5 S 39$ & $\begin{array}{l}\text { 5'-CCATTGTATTAGGGTCTCCAG-3' } \\
\text { 5'-CTCTTGGTTCCTGGCTTCGG-3' }^{\prime}\end{array}$ & $60^{\circ} \mathrm{C}$ & $1 \cdot 0$ & 240 \\
\hline D5S127 & $\begin{array}{l}5^{\prime} \text {-CTGGGTTGCAGATGGCTACCTTC-3' } \\
\text { 5'-GTACCCTTACAGGAAGAGCCAAG-3' }^{\prime}\end{array}$ & $62^{\circ} \mathrm{C}$ & 1.0 & 120 \\
\hline
\end{tabular}

PCR conditions for a $6 \mu \mathrm{l}$ reaction: $50 \mathrm{mmol} / 1 \mathrm{KCl}, 10 \mathrm{mmol} / 1$ tris- $\mathrm{HCl}, 0.01 \%$ glycerol, $1 \mathrm{pmol}$ of each primer, $200 \mu \mathrm{mol} / 1 \mathrm{dCTP}$ dGTP, dTTP, $25 \mu \mathrm{mol} / 1$ dATP, $0.2 \mu l\left[{ }^{35} \mathrm{~S}\right] \mathrm{dATP} \alpha \mathrm{S}(>1000 \mathrm{Ci} / \mathrm{mmol}$, Amersham), 0.15 units Taq DNA polymerase (PerkinElmer), 2-10 ng of template DNA, and the specified concentration of $\mathrm{MgCl}_{2}$. The reactions were denatured at $94^{\circ} \mathrm{C}$ for one minute, annealed for one minute at the specified temperature, and extended at $72^{8} \mathrm{C}$ for one minute (extension step may be omitted for JK53CA1/2) for 35 cycles, with a final extension at $72^{\circ}$ for four minutes. Half the reaction volume was electrophoresed in a $6 \%$ denaturing polyacrylamide gel (with formamide loading buffer) at $50 \mathrm{~W}$ for the specified time. 


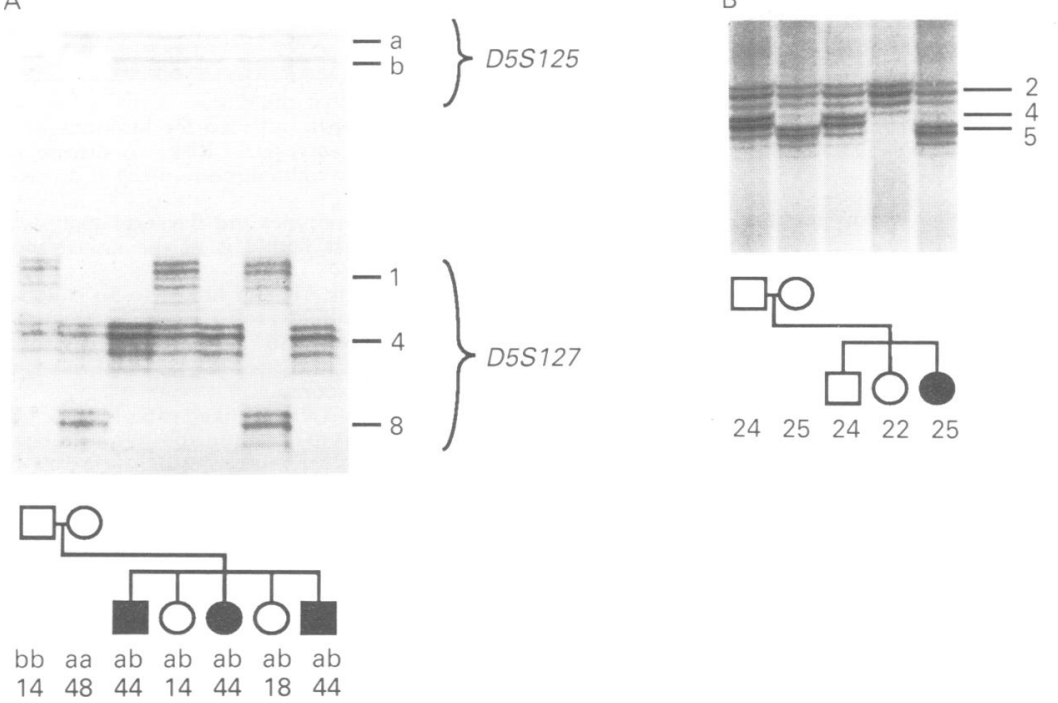

C

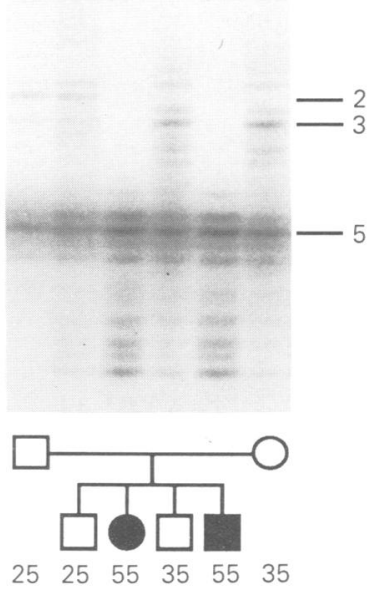

Figure 1 Segregation of $(C A)$ dinucleotide repeat polymorphisms in three SMA families at D5S125 and D5S127 (A),D5S39 (B), and fK $53 C A 1 / 2(C)$.

Table 3 Two point lod scores for D5S127 and loci at 5q11-q13.

\begin{tabular}{lrrrrrrrr}
\hline \multicolumn{8}{c}{ Recombination fractions } \\
& 0.001 & 0.01 & 0.05 & 0.1 & 0.2 & 0.3 & $\mathrm{Z}_{\max }$ & $\theta$ \\
\hline DSS127 v & & & & & & & & \\
DSS6 & 1.43 & 6.08 & 7.98 & 7.51 & 5.20 & 2.69 & 7.99 & 0.05 \\
SMA & -5.39 & 1.35 & 5.05 & 5.64 & 4.68 & 2.94 & 5.64 & 0.10 \\
DSS125 & 7.27 & 7.09 & 6.32 & 5.35 & 3.46 & 1.78 & 7.29 & 0.00 \\
fKS 53CA1/2 & 14.60 & 15.25 & 14.36 & 12.63 & 8.82 & 5.01 & 15.25 & 0.01 \\
DSS39 & 14.46 & 14.30 & 13.30 & 11.81 & 8.52 & 5.14 & 14.48 & 0.00 \\
\hline
\end{tabular}

Family 1 Severe SMA

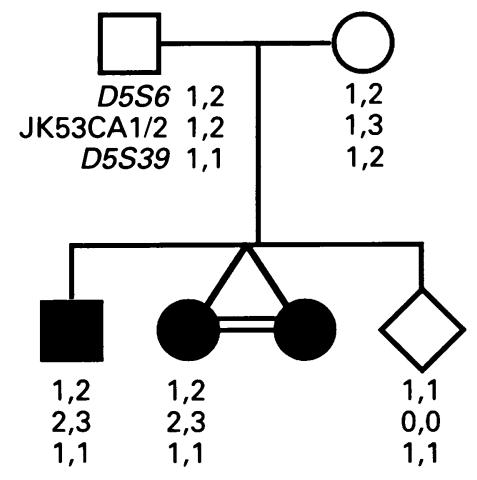

Family 3 Intermediate SMA

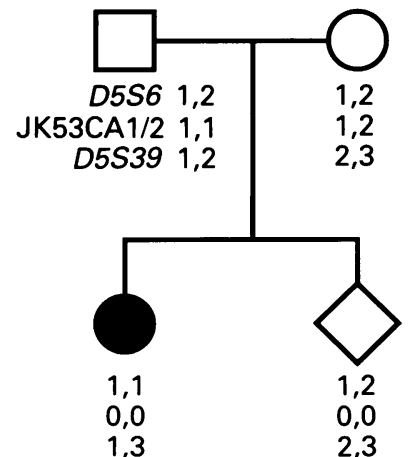

Family 2 Severe SMA

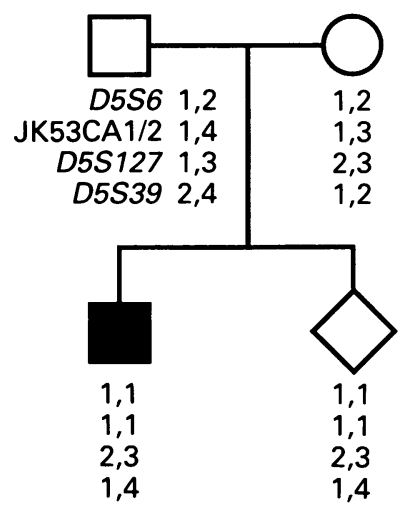

Family 4 Intermediate SMA

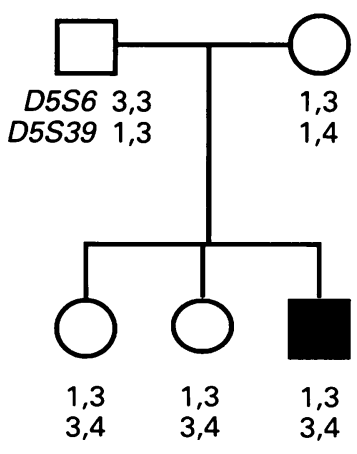

The mode of inheritance was assumed to be autosomal recessive. The prior probability that the family was of the linked type was $95 \%$. As there was only one child in the sibship, this probability could not be altered by evaluation of genotypes within the family.

After chorionic villus sampling at 12 weeks' gestation, the fetal genotype was determined at D5S6, $\mathcal{F K} 53 C A 1 / 2$, D5S127, and D5S39. Genotyping at D5S125 was not successful. Assuming the family to be of the linked type, the risk of the fetus being affected was then estimated for four locations of $S M A$ within the interval D5S6-( $F K 53 C A 1 / 2, D 5 S 112)$. The risk was greater than $99 \%$. Assuming the family to be of the unlinked type, the risk of the fetus being affected was $25 \%$. The final weighted risk of the fetus being affected was $96 \%$. The pregnancy was terminated.

Family 3. Intermediate SMA (fig 2)

The proband was diagnosed at 17 months of age. His development had been normal during the first few months of life, but weakness was noted from the age of 9 months. He sat unaided at 11 months, but never stood or walked alone. The clinical findings, muscle ultrasound, and a muscle biopsy were consistent with a diagnosis of intermediate SMA. The proband's parents presented during the second pregnancy requesting prenatal diagnosis.

The empirical recurrence risk in this situation is approximately $20 \%,{ }^{9}$ which presumably reflects autosomal recessive inheritance together with a proportion of misdiagnoses, new mutations, or non-genetic causes. If the diagnosis of SMA is correct, the penetrance of autosomal recessive SMA is 1.0 , and autosomal dominant and $\mathrm{X}$ linked forms of the disease have been excluded, the prior probability of an isolated case of intermediate SMA being autosomal recessive is approximately $80 \%$, the remainder of the risk being attributable to non-inherited causes. Although these assumptions appear demanding, the fact that linkage heterogeneity has not been documented in

Figure 2 Pedigrees and genotypes of four SMA families discussed in the text. 
Table 4 Prenatal diagnostic protocol for autosomal recessive SMA.

\begin{tabular}{|c|c|c|c|}
\hline & Diagnosis in proband* & Sibs & Course of action \\
\hline $1 \cdot 1$ & Severe SMA & None & $\begin{array}{l}\text { AR† inheritance. Probability that the disease locus is linked to } 5 \mathrm{q} \text { is } \\
95 \% \text {. Estimate fetal risk of being affected for locations of } S M A \text { in } \\
D 5 S 6-(\mathcal{Y} K 53 C A 1 / 2, D 5 S 112) \text { interval. } \ddagger \text { Risk if disease locus is } \\
\text { unlinked is } 25 \% \text {. Final risk is weighted mean of risk if disease locus is } \\
\text { linked and risk if unlinked. }\end{array}$ \\
\hline $1 \cdot 2$ & & Any number & $\begin{array}{l}\text { AR inheritance. Use family genotypes and Bayesian analysis to deter- } \\
\text { mine posterior probability that family is of the linked type. Then } \\
\text { proceed as in protocol } 1 \cdot 1 \text {. }\end{array}$ \\
\hline $2 \cdot 1$ & Intermediate SMA & None & $\begin{array}{l}\text { Prior probability that disease is } \mathrm{AR} \text { and linked to } 5 \mathrm{q} \text { is } 80 \% \text {. Ignore } \\
\text { possibility of AR inheritance unlinked to } 5 \mathrm{q} \text {. Estimate fetal risk of being } \\
\text { affected for locations of } S M A \text { in } D 5 S 6-(\Im K 53 C A 1 / 2, D 5 S 112) \text { interval. } \\
\text { Assume recurrence risk in sporadic cases is } 1 \% \text {. Final risk is weighted } \\
\text { mean of risk if AR and risk if sporadic. }\end{array}$ \\
\hline $2 \cdot 2$ & & Any number & $\begin{array}{l}\text { Prior probability that disease is } A R \text { and linked to } 5 \mathrm{q} \text { is } 80 \% \text {. Use family } \\
\text { genotypes and Bayesian analysis to determine posterior probability that } \\
\text { disease is AR. Then proceed as for protocol } 2 \cdot 1 \text {. }\end{array}$ \\
\hline $\begin{array}{l}3 \cdot 1 \\
3 \cdot 2\end{array}$ & Mild SMA & $\begin{array}{l}\text { None } \\
\text { Any number }\end{array}$ & $\begin{array}{l}\text { Prior probability that disease is AR is } 40 \% \text {. Proceed as for protocol } 2 \cdot 1 \text {. } \\
\text { Prior probability that disease is AR is } 40 \% \text {. Proceed as for protocol } 2 \cdot 2 \text {. }\end{array}$ \\
\hline
\end{tabular}

* Autosomal dominant SMA, $\mathrm{X}$ linked SMA, the various distal SMA syndromes, and $\mathrm{X}$ linked muscular dystrophies must be excluded before considering prenatal diagnosis.

$\dagger \mathrm{AR}=$ autosomal recessive.

The genetic map is cen-D5S6-[0.02]-D5S125-[0.04]-( $f K 53 C A 1 / 2, D 5 S 112)-[0 \cdot 04]-(D 5 S 127, D 5 S 39)$-qter, with $S M A$ located between $D 5 S 6$ and $(\Im K 53 C A 1 / 2, D 5 S 112)$.

familial intermediate SMA despite assuming complete penetrance provides support for this line of reasoning. As the proband did not have any sibs, the prior probability could not be altered by evaluation of genotypes within the family. After chorionic villus sampling at 10 weeks' gestation, the fetal genotypes at D5S6 and D5S39 were determined. Assuming that the disease locus in this family was autosomal recessive and linked to $5 \mathrm{q}$ loci, the risk of the fetus being affected was less than $2 \%$. Because the prior probability of the disease locus being autosomal recessive but unlinked to $5 \mathrm{q}$ loci is small relative to the prior probability of the disease being non-inherited, the recurrence risk attributable to an autosomal recessive unlinked locus was ignored. The prior probability of intermediate SMA being non-inherited in this family is approximately $20 \%$; the recurrence risk here was arbitrarily taken to be $1 \%$. Therefore the final weighted risk of the fetus having intermediate SMA was approximately $1 \cdot 8 \%$. The pregnancy proceeded to a normal delivery at term; in the immediate postnatal period the baby appeared normal.

\section{Family 4. Intermediate SMA (fig 2)}

The proband was noted to be hypotonic at 5 months of age. He sat unaided but unsteadily at 12 months of age; stable sitting was achieved at 18 months of age. He never stood or walked unaided. The clinical findings, EMG, and muscle biopsy were consistent with a diagnosis of intermediate SMA. He had two unaffected sisters.

Although the parents have not presented requesting prenatal diagnosis, the following protocol would be appropriate. The prior probability that the disease is autosomal recessive is $80 \%$. The proband and his two sisters had the same genotypes at D5S6 and D5S39, suggesting that SMA was not autosomal recessive in this family. On the basis of the genotypes at D5S6 and D5S39, the likelihood of the disease being a new mutation rather than autosomal recessive was $0 \cdot 71$. (The relative likelihood of observing these genotypes was calculated assuming SMA to be autosomal recessive and located at DSS125 (versus all loci being unlinked). The proband was then coded as normal, thereby mimicking a new mutation, and the relative likelihood recalculated. The difference in the two relative likelihoods was the likelihood that the disease was autosomal recessive rather than noninherited.) Therefore the posterior probability that the disease was autosomal recessive was $62 \%$. In a subsequent pregnancy the risk of the fetus being affected could be calculated assuming autosomal recessive inheritance and using the fetal genotypes at D5S6 and D5S39. If the proband had a non-inherited form of SMA the recurrence risk would be $1 \%$. The two risks could be weighted according to the posterior probabilities that the disease was autosomal recessive or non-inherited in this family.

\section{Discussion}

The disease locus in families with autosomal recessive SMA has been mapped to 5q11-q13 in pedigrees having two or more affected members. ${ }^{1-578}$ We have genetically mapped a series of highly polymorphic loci that are very close to $S M A$. The order of loci is cen-D5S6-(SMA,D5S125)-( $\mathcal{F}$ K3CA1/2, D5S112)-(D5S127,D5S39)-qter. The genotypes at all loci other than D5S6 and D5S112 can be determined from dried blood spots, and hence genotypic information can be obtained from the Guthrie card of a child who has died. Therefore, the stage should be set for the provision of prompt and accurate prenatal diagnosis of this often distressing disease.

The problems of potential misdiagnosis, uncertain mode of inheritance, and linkage heterogeneity in spinal muscular atrophy should make one cautious in applying this genetic map in a counselling situation. Nevertheless, it is possible to use this genetic map as the basis for prenatal diagnosis. Our current approach to prenatal testing of autosomal recessive SMA is summarised in table 4.

We would emphasise that any approach to prenatal diagnosis must be based on a correct diagnosis in the proband. Because of the wide phenotypic variation in SMA, the diagnosis is not always straightforward. Where there is 
doubt, appropriate consultation should be obtained before proceeding with prenatal diagnosis. It should also be noted that the disease locus in autosomal dominant SMA is not localised to 5q11-q13. ${ }^{26}$ Although we have not documented linkage heterogeneity in our set of 31 pedigrees with two or more affected subjects, we consider it prudent to assume some degree of linkage heterogeneity (or possible misdiagnoses) when estimating risks in small families with severe SMA. Although it is arguable whether the risk in an unlinked pedigree should be $25 \%$ (that is, assuming autosomal recessive inheritance) or zero, in practice it makes little difference to the risk figure.

We have no experience of prenatal diagnosis in families with mild SMA. The empirical recurrence risk is 6 to $10 \% .^{9}$ (Empirical recurrence risk figures may be higher in some populations. ${ }^{9}$ This figure was derived from studies in the north of England and in London.) Making the same assumptions as outlined above for intermediate SMA (family 3), the prior probability of an isolated case of mild SMA being autosomal recessive is approximately $40 \%$, the remainder of the risk being attributable to non-inherited causes. As before, these assumptions appear quite demanding, especially as the proportion of non-inherited cases seems relatively high. In support of these prior probabilities is the lack of linkage heterogeneity in families with mild SMA despite the assumption of complete penetrance. We would stress that the choice of prior probabilities is used simply to partition the recurrence risk into components which can be readily estimated for a family, and do not represent a complete genetic model of the disorder. On this basis prenatal diagnosis would be possible along the lines detailed for families with intermediate SMA.

When linkage analysis is used as the basis of prenatal diagnosis, genotypes should be determined at loci which flank the disease locus. The loci D5S6 and ( $F K 53 C A 1 / 2, D 5 S 112)$ lie $6 \mathrm{cM}$ apart and flank $S M A .{ }^{8}$ RFLPs have been documented at D5S6 and D5S112 (table 1). As yet, a PCR based polymorphism has not been detected at D5S6 or at a locus tightly linked to it, and determination of the fetal genotype at D5S6 requires more placental tissue and time than determination of genotypes at other loci. If a family is uninformative at D5S6 there is no other locus that is highly informative, precisely mapped close to $S M A$, and centromeric to D5S6. Our current practice is to determine genotypes at D5S6, D5S125/D5S127 (a single PCR reaction), and $f K 53 C A 1 / 2$. The dinucleotide repeat polymorphism at D5S39 is more difficult to interpret than the polymorphism at the tightly linked locus D5S127.

Ideally, a prenatal diagnostic protocol such as we describe should be audited to determine the frequency of incorrect diagnoses of the fetus. False negative diagnoses would be readily ascertained when a supposedly homozygous normal or heterozygous child developed symptoms. On three occasions we have concluded that a fetus would be at low risk of being affected with severe or intermediate SMA; one pregnancy miscarried (family 1), one baby appears normal in the neonatal period (family 3), and non-paternity was suspected in a third instance. Documented cases of false negative diagnoses should be reported promptly. False positive diagnoses are more of a problem. It is often difficult to obtain fetal tissues suitable for histopathological examination during a termination of pregnancy at 10 to 12 weeks' gestation. Even if such tissues were obtained, there are no recognised pathological features that are specific for SMA at this gestation.

A three allele model for SMA could have significant implications for prenatal diagnosis and counselling. ${ }^{13}$ According to this model, the alleles at SMA may be normal $(+)$, mutant $(-)$, or 'activator' (a) with allele frequencies of $0.8999,0.0001$, and $0 \cdot 1000$, respectively. Only the genotypes $-/-$ and $a /-$ result in the SMA phenotype. If this model is correct, one of the parents of an affected subject could have an a/a genotype. In this situation the recurrence risk would be $50 \%$, and prenatal diagnosis based on an autosomal recessive model would be liable to error. It has been suggested that prenatal diagnosis in SMA be deferred until this is resolved. However, this approach is unnecessarily cautious. Under this model, the only joint parental genotype that could give a $50 \%$ recurrence risk in a sibship is $+/-, \mathbf{a} / \mathbf{a}$ (assuming the parents are unaffected). All other parental genotype combinations would result in either no affected children or in a recurrence risk of $25 \%$ in the sibship. The probability that the parents of a single affected child have a joint genotype of $+/-, a / a$ compared to genotype combinations with lower risks is only $10 \%$. There would be insufficient information in a small pedigree to determine whether the two or three allele model is more likely to be correct and hence to weight the risk figures derived under each model. In practice it may be worth calculating the relative likelihoods of the two versus three allele models when advising large pedigrees with affected second and third degree relatives. The risks estimated under each model could then be weighted according to the relative likelihoods.

We would like to thank Drs John Tolmie, Howard Hughes, and J Ignatius for referring families; Dr John Old for technical assistance with CVS samples; Dr Anthony Monaco for access to his YAC library; and Sue Clark for technical assistance. This work was funded by the Medical Research Council, the Muscular Dystrophy Association, USA, the Muscular Dystrophy Group of Great Britain and Northern Ireland, and the Nuffield Foundation.

1 Brzustowicz LM, Lehner T, Castilla LH, et al. rzustowicz LM, Lehner T, Castilla LH, et al. Genetic mapping of chronic childhood-onset spinal muscular atrophy to chromosome 5q11.2-13.3. Nature 1990;344:540-1.

2 Gilliam TC, Brzustowicz LM, Castilla LH, et al. Genetic homogeneity between acute and chronic forms of spinal muscular atrophy. Nature 1990;345:823-5.

3 Melki J, Abdelhak S, Sheth P, et al. Gene for chronic proximal spinal muscular atrophies maps to chromosome 5q. Nature 1990;344:767-8.

4 Melki J, Sheth P, Abdelhak S, et al. Mapping of acute (type 1) spinal muscular atrophy to chromosome 5q12-q14. Lancet 1990;336:271-3.

5 Sheth P, Abdelhak S, Bachelot MF, et al. Linkage analysis in spinal muscular atrophy, by six closely flanking 
markers on chromosome 5. Am $\mathcal{f}$ Hum Genet 1991;48:764-8.

6 Dubowitz V. Muscle disorders in childhood. Philadelphia: Saunders, 1978:146-78.

7 Daniels RJ, Thomas NH, MacKinnon RN, et al. Linkage analysis of spinal muscular atrophy. Genomics (in press) analysis of spinal muscular atrophy. Genomics (in press).
Morrison KE, Daniels RJ, Suthers GK, et al. High resolu-

8 Morrison KE, Daniels RJ, Suthers GK, et al. High resolution genetic map around the SMA locus chromosome 5. Am $\mathcal{F}$ Hum Genet (in press).

9 Bundey S. Genetics and neurology. Edinburgh: ChurchillLivingstone, 1985.

10 Baraitser M. The genetics of neurological disorders. Oxford Oxford University Press, 1982.

11 Becker PE. Atrophia musculorum spinalis pseudomyopathica. Z Menschl Vererb u Konstitutionslehre 1964; 37:193-220.

12 Bouwsma G, Leschot NJ. Unusual pedigree patterns in seven families with spinal muscular atrophy; further evidence for the allelic model hypothesis. Clin Genet 1986;30:145-9.

13 Muller B, Clerget-Darpoux F. Becker's model and prenatal diagnosis in proximal spinal muscular atrophy (SMA): a diagnosis in proximal spinal muscular atrophy (SMA)

14 Munsat TL, Skerry L, Korf B, et al. Phenotypic heterogeneity of spinal muscular atrophy mapping to chromosome 990;40:1831-6.

15 Dubowitz V. Chaos in classification of the spinal muscular atrophies of childhood. Neuromuscular Disorders 1991;1:77-80.

16 Weber JL. Informativeness of human (dC-dA)n.(dG-dT)n polymorphisms. Genomics 1990;7:524-30.
17 Kidd KK, Bowcock AM, Schmidtke K, et al. Report of the DNA committee and catalogs of cloned and mapped DNA committee and catalogs of cloned and mapped
genes and DNA polymorphisms. HGM10. Cytogenet Cell genes and DNA polymor
Genet 1989;51:622-947.

18 Sherrington R, Mankoo B, Dixon M, et al. Microsatellite polymorphisms provide a highly polymorphic map for chromosome 5 bands q11.2-q13.3. Genomics (submitted).

19 Williamson R, Bowcock AM, Kidd K, et al. Report of the DNA committee and catalogues of cloned and mapped genes and DNA polymorphisms. HGM10.5. Cytogenet Cell Genet 1990;55:457-778.

20 Mankoo BS, Sherrington R, De la Concha A, et al. Two microsatellite polymorphisms at the D5S39 locus. Nucleic Acids Res 1990;19:1963.

21 Lathrop GM, Lalouel JM, Julier C, Ott J. Strategies for multilocus linkage analysis in humans. Proc Natl Acad Sci USA 1984;81:3443-6.

22 Weeks DE, Ott J. Risk calculations under heterogeneity. Am f Hum Genet 1989;45:819-21.

23 Narod S. Counselling under genetic heterogeneity. Clin Genet 1991;39:125-31.

24 Krawczak $M$. Genetic risk and recombination fraction - an example of non-monotonic dependency. Hum Genet example of no

25 Lange K. Approximate confidence intervals for risk prediction in genetic counselling. Am $\mathcal{f}$ Hum Genet 1986;38:681-7.

26 Kausch K, Muller CR, Grimm T, et al. No evidence for linkage of autosomal dominant proximal spinal muscular atrophies to chromosome 5q markers. Hum Genet 1991;86:317-8. 